\section{FERTILITÄTSPROBLEME}

\section{Gesund ernährt - bessere Spermien}

Mit Vollkornprodukten, Obst und Gemüse, dafür weniger Transfettsäuren lässt sich offenbar die Spermienqualität deutlich verbessern. Dr. Audrey Gaskins aus Boston hatte mit seinem Team die Qualität der Spermien bei 188 jungen Männern untersucht und mit Angaben zu ihrer Ernährung verglichen. Sie unterschieden zwischen einer "westlichen" Ernährung mit viel Fleisch, Wurst, Pizza, Weißmehl, Zucker und Limonade und einer „umsichtigen“ Ernährung mit viel Fisch, Obst und Vollkornprodukten.

Männer, die sich besonders stark an die westliche Diät hielten, hatten zwar eine höhere Spermienkonzentration, dafür war die Motilität der Spermien in der Gruppe der Männer, die sich besonders stark an die umsichtige Diät hielten, besser. So waren in dem Quartil der Männer mit der gesündesten Ernährungsweise $63 \%$ der Spermien beweglich, aber nur $56 \%$ in dem Quartil der "ungesunden“ Esser. Man vermutet, dass der geringere Anteil an Transfettsäuren in der gesunden Ernährung hierfür verantwortlich ist.

Fertility and Sterility 2011; 90 Suppl O-27, 0-48 http://www.fertstert.org/supplements

\title{
ERHÖHTES KREBSRISIKO
}

\section{Grillsteaks lassen Darmpolypen gedeihen}

Personen, die viel Fleisch essen, haben häufiger kolorektale Polypen. Besonders ungünstig ist der Verzehr von rotem Fleisch, das stark erhitzt, also gebraten oder gegrillt wurde. Das hat die bisher größte Koloskopiestudie zu diesem Thema bestätigt.

An der Studie beteiligten sich 2543 Patienten mit Darmpolypen, davon 1881 mit Adenomen und 622 mit hyperplastischen Polypen (HPP), sowie 3764 Personen, bei denen kein Polyp entdeckt worden war. Ein erhöhtes Risiko für Adenome und HPP fand sich bei ausgeprägten Karnivoren mit Vorliebe für rotes und/oder verarbeitetes Fleisch (Schinken, Wurst). Der Risikoanstieg konnte auf Fleischmahlzeiten aus der Pfanne oder vom Grill zurückgeführt werden. Mit niedrigeren Temperaturen zubereitetes rotes Fleisch hatte keinen Einfluss auf das Vorkommen von Darmpolypen. Reichlicher Verzehr von weißem Fleisch, sprich Huhn oder Fisch, war nicht mit einem höheren Risiko für Polypen assoziiert.

Cancer Prev Res 2011; 4: 1686-97

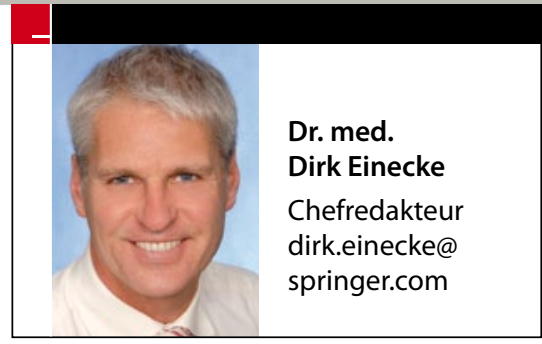

LUNGENKREBSSCREENING Jährliches Röntgen senkt Mortalität nicht

Für ein Screening auf Bronchialkarzinome sind Röntgen-Thorax-Untersuchungen nicht geeignet, belegt eine randomisierte Studie mit über 150000 Teilnehmern. Der Hälfte von ihnen wurde vier Jahre lang jährlich eine Röntgen-Thorax-Untersuchung angeboten. Die übrigen Studienteilnehmer erhielten nur die übliche medizinische Versorgung. Die Nachbeobachtungszeit betrug maximal 13 (im Median knapp 12) Jahre. In dieser Zeit wurden in beiden Gruppen gleich häufig Bronchialkarzinome diagnostiziert. Nur 307 von 1696 Bronchialkarzinomen in der Interventionsgruppe (18\%) wurden durch das Screening zutage gefördert. Die Krebsdiagnose erfolgte nicht in früheren Stadien als in der Vergleichsgruppe. Bei 12718 Teilnehmern lieferte mindestens eine Screeninguntersuchung ein falsch-positives Ergebnis. Die Abklärung war bei $0,4 \%$ von ihnen mit Komplikationen belastet. In der Interventionsgruppe starben 1213 Teilnehmer an Lungenkrebs, in der Kontrollgruppe waren es 1230.

JAMA 2011; doi:10.1001/jama.2011.1591

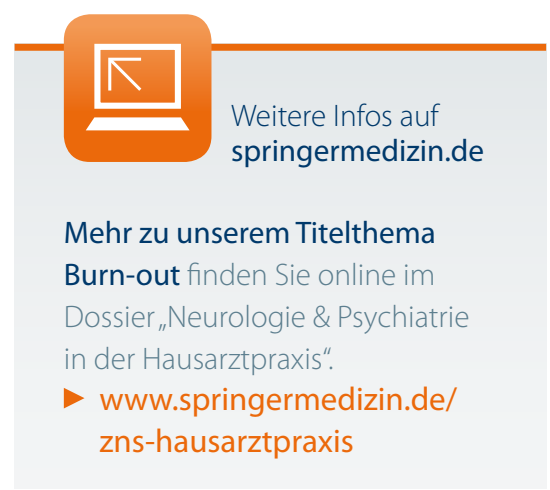

\section{PROSTATAKREBS}

\section{Mit PSA-Screening lebt keiner länger}

Bei Männern, die regelmäßig ihren PSAWert bestimmen lassen, wird ein Prostatakarzinom zwar früher erkannt, sie leben aber nicht länger als diejenigen, die sich die Vorsorgeuntersuchungen sparen. Dies zeigt ein großes Review der U.S. Preventive Services Task Forces (USPSTF). Zwar wurden mit dem PSA-Screening tatsächlich mehr Karzinome früh diagnostiziert, aber auf die krebs- spezifische Mortalität innerhalb von zehn Jahren hatte das keinen Einfluss. Bei 12-13\% der gescreenten Männer war das Ergebnis falsch-positiv. 0,5-1\% der darauf durchgeführten Biopsien hatten Infektionen oder Harnverhalt zur Folge. Auch werden viele Low-Risk-Tumoren angezeigt, die den Patienten nie Probleme gemacht hätten.

Ann Intern Med, first published October 7, 2011. 